\title{
Cicatrización normal y patológica: una revisión actual de la fisiopatología y el manejo terapéutico
}

\author{
Current concepts of the approach and treatment of normal and \\ pathological wound healing: a literature review
}

\author{
Carlos Altamirano Arcos'1, Rogelio Martínez Wagner², Enrique Chávez Serna ${ }^{3}$, \\ Christian Altamirano Arcos ${ }^{4}$, Israel Espino Gaucin ${ }^{1}$, Luciano Nahas Combina ${ }^{1}$
}

\section{RESUMEN}

El conocimiento del proceso de cicatrización normal y patológica es fundamental para todas las especialidades médicas y quirúrgicas que tratan heridas agudas y crónicas, ya que del efecto de estos procesos dependerá el resultado final del tratamiento quirúrgico.

En cada tejido y órgano dentro del organismo existen diferentes mecanismos que regulan la función y homeostasis celular, como sucede en el proceso de cicatrización, en donde participan y contribuyen una serie de fases y factores mediados por células y señales químicas. Una cicatrización aceptable es aquella que deja una adecuada cicatriz externa, devuelve la normalidad anatómica y funcional del tejido, con lo que se espera llegar a tener un resultado final con éxito; Sin embargo se debe entender que este proceso es complejo, y pueden también existir variantes anormales, determinadas por ciertos factores que intervienen para dar resultado a una cicatriz patológica, para lo cual existen diferentes tipos de tratamientos específicos y coadyuvantes para cada una de ellas.

Objetivo: Conocer los conceptos actuales en el abordaje y tratamiento de la cicatrización normal y patológica, ofreciendo al cirujano una guía práctica basada en los fundamentos de las investigaciones científicas actuales.

Material y métodos: Se realizó una revisión bibliográfica de artículos recientes acerca de cicatrización publicados entre 2010 a 2018, con alto nivel de evidencia.

Palabras claves: cicatrización normal, cicatrización patológica, diagnóstico, tratamiento, abordaje, complicaciones.

\begin{abstract}
The knowledge of normal and pathological wound healing process is essential for all the medical and surgical specialties that treat acute and chronic wounds, because the final result will depend on the effect of these processes.

In each tissue and organ within the organism there are different mechanisms that regulate cellular function and homeostasis, as in the wound healing process, where a series of phases and factors mediated by cells and chemical signals participate. Acceptable wound healing, although it leaves an external scar, restores the anatomical and functional homeostasis of the tissue,which is expected to have a successful result; However, it must be understood that this is a complex process, and therefore may also exist abnormal variants, determined by certain factors that lead to pathological wound healing, hence different types of treatments and coadjuvants therapies are available.

Objective: Comprehend the current concepts in the approach and treatment of normal and pathological wound healing, offering the surgeon a practical guide based on state of the art evidence,

Material and Methods: A literature review of recent articles published between 2010 and 2018 was carried out, with a high level of evidence.
\end{abstract}

Keywords: normal wound healing, pathological wound healing, diagno sis, treatment, approach, complications.

REVISTA ARGENTINA DE CIRUGÍA PLÁSTICA 2019;25(1):11-15. DOI/10.32825/RACP/201901/0011-0015

\section{INTRODUCCIÓN}

El conocimiento del proceso de cicatrización normal y patológica es fundamental para todas las especialidades médicas y quirúrgicas que tratan heridas agudas y crónicas, ya que del efecto de estos procesos dependerá el resultado final del tratamiento quirúrgico. La descripción histórica del manejo de la ci-

1. Residente de tercer año de Cirugía Plástica y Reconstructiva, Hospital General Dr. Manuel Gea González. Universidad Nacional Autónoma de México. Ciudad de México, México

2. Médico adscrito de Cirugía Plástica y Reconstructiva. Hospital General Dr. Manuel Gea González. Universidad Nacional Autónoma de México. Ciudad de México, México

3. Médico pasante de Cirugía Plástica y Reconstructiva. Hospital General Dr. Manuel Gea González. Universidad Nacional Autónoma de México. Ciudad de México, México

4. Médico general. Facultad de Ciencias Médicas Universidad de las Américas. Quito, Ecuador

$\bowtie$ Correspondencia: Dr. Carlos Altamirano Arcos. carlosalt2@gmail.com

Los autores declaran no tener conflictos de intereses. catrización se remonta al antiguo Egipto, con el documento conocido como el Papiro de Smith, desde el año 1400 a. C. Desde entonces, ha existido una comprensión cada vez mayor de la fisiología y fisiopatología de la cicatrización de heridas. Se ha logrado comprender mejor los múltiples factores intrínsecos, extrínsecos y su interacción, lo cual permite mejorar el diagnóstico de las heridas, sus etiologías y sus tratamientos. Sin embargo, a pesar de los avances en el entendimiento, la técnica y la tecnología, la proporción de heridas con cicatrización patológica continúa siendo alta.

\section{CICATRIZACIÓN NORMAL DE LAS HERIDAS}

Históricamente, la cicatrización de heridas ha sido dividida en tres fases, y algunos autores han añadido la hemostasia como la fase inicial. El proceso de cicatrización de heridas ocurre de manera continua e interpuesta y su división en fases permite una fácil descripción y evaluación. Cada fase es crítica para el éxito del cierre 
de la herida, y las alteraciones en este proceso se asocian con retrasos o cicatrización patológica.

\section{FASES DE LA CICATRIZACIÓN}

Las fases que componen el proceso de cicatrización son:

- Fase de hemostasia.

- Fase inflamatoria.

- Fase de proliferación.

- Fase de maduración y remodelación.

\section{FACTORES QUE INTERVIENEN EN EL PROCESO DE CICATRIZACIÓN}

\section{ISQUEMIA E HIPOXIA}

El proceso de cicatrización de una herida demanda energía. Los neutrófilos y los fibroblastos dejan de funcionar apropiadamente con bajos niveles de oxígeno. Las reducciones en la tensión de oxígeno se correlacionan con resultados desfavorables. La disminución de la tensión subcutánea del oxígeno resulta en tasas más altas de infección de la herida, y el depósito de colágeno está directamente relacionado con la tensión del oxígeno de la herida y la perfusión tisular. La clave del suministro de oxígeno y nutrientes a la herida es la presión arterial parcial del oxígeno, no el contenido de oxígeno, que a menudo puede mantenerse por medio de modificaciones en la vasodilatación, el gasto cardíaco y la permeabilidad capilar.

\section{INFECCIÓN}

La proliferación bacteriana dentro de una herida puede resultar en alteraciones de la cicatrización. La hemostasia puede alterarse a través de efectos bacterianos sobre plaquetas y el complemento. La infección bacteriana causa aglutinación plaquetaria, trombocitopenia y periodos prolongados de inflamación que afecta la formación de tejido de granulación. Se ha evidenciado un retraso en la cicatrización a niveles por encima de $10^{3}$ bacterias por mililitro. El inóculo exacto necesario para el diagnóstico cuantitativo de la infección es de $10^{5}$ unidades formadoras de colonias por gramo de tejido para la mayoría de las bacterias.

La excepción son los estreptococos $\beta$-hemolíticos, cuya simple presencia en una herida puede indicar infección $n^{1,2}$.

\section{TABAQUISMO}

El humo del tabaco contiene más de 4000 componentes. Los que contribuyen a la alteración del proceso normal de cicatrización son: la nicotina, el monóxido de carbono y el cianuro de hidrógeno. La nicotina actúa como un vasoconstrictor, dando como resultado isquemia local, la cual puede reducir el flujo sanguíneo hasta un $40 \%$, este efecto es temporal y los niveles de oxígeno vuelven a la normalidad $45 \mathrm{mi}-$ nutos después de consumido el tabaco. El monóxido de carbono, cuenta con una afinidad de unión a la hemoglobina 200 veces mayor que la del oxígeno, produciendo carboxihemoglobina y reduciendo el aporte de oxígeno a la herida. Aunque no existe una guía uniforme para el momento de la abstinencia preoperatoria, expertos recomiendan un intervalo de 4 semanas antes y 4 semanas después de la cirugía estética o reconstructiva ${ }^{3}$.

\section{DIABETES}

La diabetes es un factor de riesgo para alteraciones en el proceso de cicatrización porque se asocia con vasculopatía, neuropatía e inmunopatía. La hiperglucemia resulta en la modificación de proteínas y enzimas a nivel de la membrana basal, alterando la permeabilidad y el suministro de nutrientes al lecho de la herida. Se produce una micro y macroangiopatía, lo que puede resultar en un suministro insuficiente de oxígeno ${ }^{4}$.

\section{DEFICIENCIA NUTRICIONAL}

El proceso normal de cicatrización se encuentra alterado cuando existe un déficit de macro y micronutrientes. La desnutrición proteica afecta gravemente este proceso principalmente con la deficiencia en los aminoácidos arginina y glutamina. Esto se debe a que la síntesis de estos aminoácidos es insuficiente durante los períodos de aumento de la utilización de proteínas que se producen durante la cicatrización de heridas. El monitoreo del estado nutricional se realiza mediante la medición de albúmina (vida media 21 días), prealbúmina (vida media 2 días) y transferrina (vida media 7 días) $)^{5,6}$.

\section{FÁRMACOS}

El consumo de esteroides sistémicos tiene un efecto negativo sobre la cicatrización de la herida. Disminuyen la inflamación, inhiben la epitelización y disminuyen la producción de colágeno. La respuesta clínica a estos efectos moleculares es un aumento en la dehiscencia de las incisiones quirúrgicas, un mayor riesgo de infecciones de la herida y un retraso en la cicatrización ${ }^{7}$.

\section{RADIACIÓN}

El efecto más importante de la radiación es su daño al $\mathrm{ADN}$, que se produce cuando las partículas crean reacciones en cadena que conducen a rupturas de una o dos hebras o ruptura completa de la doble hélice. La radiación también crea radicales libres que dañan las proteínas y las membranas celulares. Las enzimas de reparación del ADN reparan algunos de los daños, pero la agresión generalmente sobrepasa las capacidades reparadoras del cuerpo y da como resultado daño microvascular, fibrosis y atrofia ${ }^{8}$.

\section{CICATRIZACIÓN PATOLÓGICADELASHERIDAS}

La cicatrización patológica está determinada por una prolongación de las fases normales de la cicatrización 
TABLA 1. Clasificación de las cicatrices.

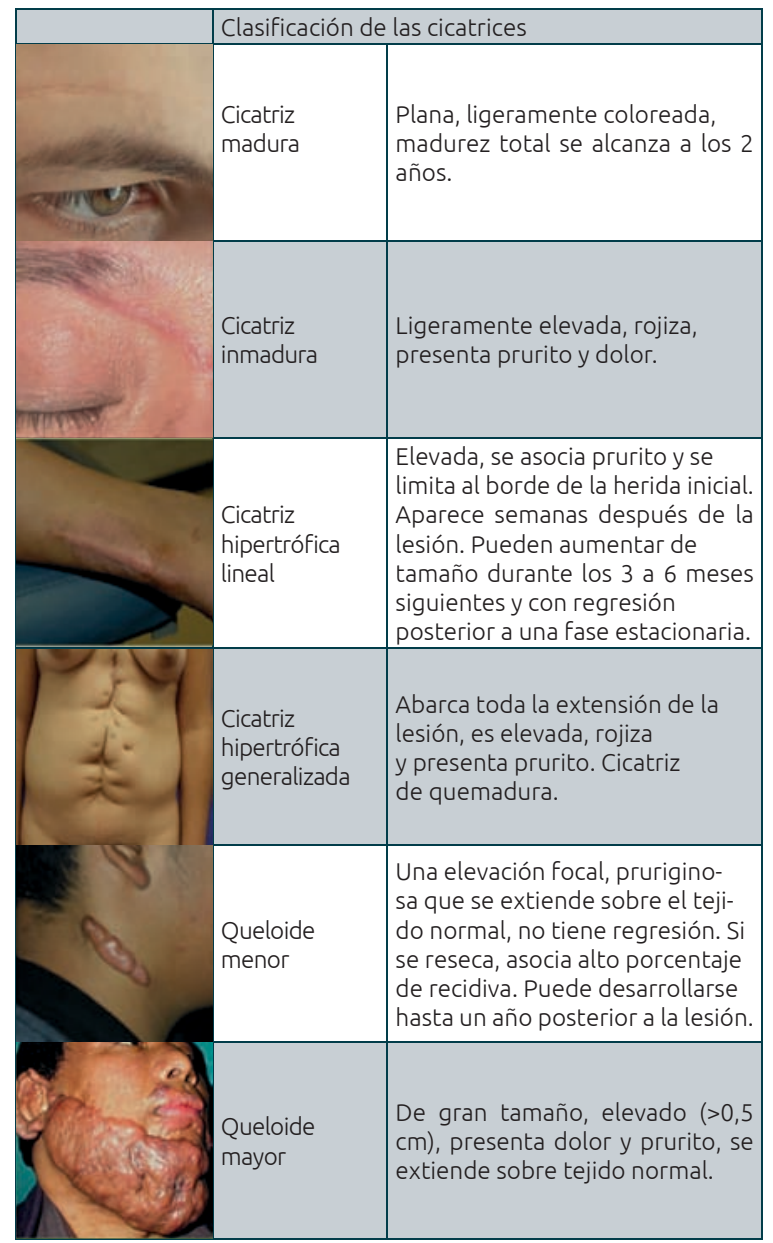

en las que se presentan alteraciones en los procesos que detienen la respuesta tisular a una lesión.

Las cicatrices hipertróficas y cicatrices queloides representan una excesiva respuesta tisular a la lesión dérmica caracterizada por la proliferación local de fibroblastos y la sobreproducción de colágeno. Los queloides (derivado de la palabra griega chele que significa "pata de cangrejo") son crecimientos fibrosos que se extienden más allá del área original de la lesión e involucran a la piel normal adyacente. Las cicatrices hipertróficas pueden tener un aspecto clínico similar, pero en contraste con los queloides, permanecen confinados dentro de los límites del área de la herida ${ }^{9,10}$.

\section{CLASIFICACIÓN}

Dentro de la clasificación descriptiva de las cicatrices, estas pueden dividirse de la siguiente manera ${ }^{11,12}$.

\section{TRATAMIENTO}

Aunque se han utilizado múltiples terapias médicas y quirúrgicas para el tratamiento de queloides y cicatrices hipertróficas, no existe en la actualidad un enfoque de tratamiento universalmente aceptado, por lo que el papel fundamental del cirujano es promover la prevención de su desarrollo ${ }^{13}$.

\section{PREVENCIÓN}

1. Mínima tensión y eversión de bordes. Una técnica quirúrgica adecuada reduce el ancho de la cicatriz y la hipertrofia. Esto incluye un cierre meticuloso con mínima tensión y con eversión del borde de la herida. La tensión provoca producción de colágeno abundante conduciendo a hipertrofia de la cicatriz. Por lo tanto, es importante el cierre por planos de la herida, aplicando la tensión a la capa fascial subyacente en lugar de la piel. La eversión de la herida se logra usando suturas dérmicas profundas ${ }^{14}$.

2. Alineación con las líneas de máxima extensibilidad y mínima tensión y respeto de las unidades anatómicas. La cirugía debe ser planeada de modo que la cicatriz coincida o sea paralela con las líneas de expresión o los pliegues cutáneos vecinos y siempre que sea posible se oculte en los márgenes de las unidades estéticas. Antes de realizar cualquier procedimiento invasivo de revisión de una cicatriz se debe esperar a que el proceso de cicatrización normal finalice. Por lo tanto, la revisión quirúrgica de la cicatriz debe ser retrasada hasta los 12-18 meses.

3. Evitar el estiramiento de la herida. Para limitar el estiramiento de la piel durante la cicatrización y facilitar el descanso apropiado de las heridas, estas pueden cubrirse con materiales protectores, como cintas, vendajes, prendas o láminas de gel de silicón.

La mayoría de los autores recomiendan iniciar la aplicación los parches de silicón a la segunda semana después de la cirugía y continuarla por lo menos 3 meses. Múltiples estudios han demostrado que el uso de silicón, en cualquiera de sus formas, sobre heridas, reduce la incidencia de cicatrización hipertrófica y además es eficaz en el tratamiento de cicatrices hipertróficas establecidas. Se prefiere el gel de silicona debido a la facilidad de aplicación ${ }^{15}$.

4. Extracto de cebolla. El componente principal de los geles de extracto de cebolla disponibles en el mercado (Mederma, Contractubex) es la quercetina extraída de la cepa Allium. Estos geles contienen además del extracto de cebolla, alantoina y heparina. La mayoría de los estudios sobre el extracto de cebolla recomiendan aplicar el ungüento 3 veces al día comenzando alrededor de una semana después de la operación, hasta 12 semanas. Este componente se ha evaluado en múltiples estudios, los cuales han encontrado evidencia contradictoria. El mecanismo de acción del extracto de cebolla no está claro. In vitro, se ha encontrado que tiene un efecto inhibidor dependiente de la dosis sobre la proliferación de fibroblastos, inhibición de histamina y regulación positiva de la metaloproteinasa de matriz. 
5. Vitamina E. La vitamina E forma parte de la familia de tocoferoles y tocotrienoles liposolubles. Presenta un modesto efecto inhibitorio sobre la síntesis de colágeno. Sin embargo, múltiples estudios muestran que la vitamina $\mathrm{E}$, no es eficaz para la prevención de cicatrices hipertróficas y queloides e incluso podría tener efectos nocivos ${ }^{16}$.

\section{MANEJO TERAPÉUTICO}

1. Corticosteroides intralesionales. Los corticosteroides actúan inactivando el TGF- $\beta 1$, lo que conduce a la activación de la colagenasa y la degradación del colágeno. Debido a sus propiedades antiinflamatorias y vasoconstrictoras, los corticosteroides intralesionales son eficaces para reducir el dolor y el prurito. El acetato de triamcinolona intralesional es el compuesto más utilizado para las cicatrices hipertróficas y queloides La dosis habitual recomendada de triamcinolona es de 3 a 4 inyecciones de triamcinolona $(10-40 \mathrm{mg} / \mathrm{ml})\left(0,5 \mathrm{cc}\right.$ por $\left.\mathrm{cm}^{2}\right)$ cada 3 a 4 semanas ${ }^{17-19}$.

2. Masaje de la cicatriz. El masaje de cicatrices es comúnmente recomendado después de una lesión por quemaduras y como prevención para de la hipertrofia en cicatrices postquirúrgicas. La evidencia muestra que el masaje parece ser más efectivo en las cicatrices posquirúrgicas que en las cicatrices por traumatismo y quemadura. El mecanismo de acción del masaje de la cicatriz no está claro, aunque hay cierta evidencia de que la presión cíclica puede inducir la apoptosis de fibroblastos.

Recomendación: existe alguna evidencia de que el masaje de cicatrices puede prevenir y mejorar las cicatrices hipertróficas (Evidencia tipo I y II).

3. 5-fluoracilo intralesional. Este compuesto se utiliza para las cicatrices que no responden a corticosteroides intralesionales. Es un análogo de la pirimidina Su mecanismo de acción no es claro, aunque parece inducir apoptosis en fibroblastos sin producir necrosis e inhibición. Su dosis es de $50 \mathrm{mg} / \mathrm{ml}$ aplicando un volumen de $0.2-0.4 \mathrm{ml} / \mathrm{cm}^{2}$ en 7 sesiones.Los efectos adversos de la incluyen dolor e hiperpigmentación ${ }^{20,21}$.

Recomendación: eficacia alta, nivel de evidencia I.

4. Bleomicina: Es un agente antitumoral derivado de Streptomyces que induce ruptura en el ADN, dando lugar a la apoptosis celular y reduciendo la actividad de los fibroblastos. La dosis total de bleomicina debe permanecer por debajo de 9 UI para evitar riesgos de fibrosis pulmonar y cutánea ${ }^{21,22}$.

Recomendación: eficacia alta, nivel de evidencia I.

5. Prendas de presión: la presión es una modalidad de tratamiento comúnmente utilizada para las cicatrices hipertróficas y queloides. Los mecanismos de acción propuestos incluyen la reducción del edema y la apoptosis de fibroblastos inducida por hi- poxia, aunque algunos estudios han demostrado un aumento de la presión sanguínea con la terapia de presión.

Para alcanzar un resultado favorable significativo en cuanto a la cicatrización es necesario una presión de al menos $24 \mathrm{mmHg}$ y apego adecuado del paciente. Se recomienda el inicio posterior a reepitelización, con uso continuo de 8 a 24 horas por los primeros 6 meses, con una presión de 24 a 40 $\mathrm{mmHg}^{23,24}$.

Recomendación: las prendas de presión pueden ser eficaces para reducir el grosor de las cicatrices hipertróficas, pero requieren altos niveles de cumplimiento por parte del paciente (nivel de evidencia I).

6. Láser: el uso de láser para alteraciones en el proceso de cicatrización cada vez va en aumento y se ha encontrado que es beneficioso para la prevención y el tratamiento de las cicatrices hipertróficas, queloides y del acné. Estudios actuales refieren que entre todos los tipos de láser, el láser de colorante pulsado (PDL) es el que tiene mayor rendimiento tanto en la prevención como en el tratamiento de las cicatrices hipertróficas ${ }^{25,26}$.

Láser de colorante pulsado: el láser colorante pulsado (PDL: pulse dye laser) de 585 y $595 \mathrm{~nm}$ se dirige selectivamente a la oxihemoglobina. El tratamiento profiláctico de nuevas cicatrices quirúrgicas con PDL ha sido evaluado en varios estudios, y los resultados son mixtos. Los estudios sobre el tratamiento de las cicatrices hipertróficas establecidas con PDL son más concluyentes. Se ha estudiado el momento del tratamiento de la cicatriz con PDL. El PDL parece ser más eficaz en las cicatrices hipertróficas inmaduras, que todavía son hipervasculares y eritematosas, al principio de la fase de remodelación. También se han estudiado otras variables PDL, como la frecuencia y la frecuencia del tratamiento. Se ha encontrado que variaciones de 3 a 7 $\mathrm{J} / \mathrm{cm}^{2}$ alcanzan una eficacia comparable y que los tratamientos repetidos cada 6 a 8 semanas son más eficaces que un solo tratamiento. Debido a que el PDL sólo penetra cerca de $1 \mathrm{~mm}$ de profundidad, las cicatrices hipertróficas gruesas pueden no responder bien a PDL. La combinación de PDL con esteroides intralesionales o 5 -fluorouracilo dan lugar a una acción sinérgica ${ }^{25}$.

Recomendación: el PDL es eficaz en el tratamiento de cicatrices establecidas (nivel de evidencia I). El láser de $\mathrm{CO}^{2}$, con su penetración más profunda, es el más adecuado para el tratamiento del contorno irregular de la cicatriz (nivel de evidencia II) ${ }^{26,27}$.

7. Cámara hiperbárica: el tratamiento con oxígeno hiperbárico es un tratamiento adyuvante en la cicatrización que aporta $\mathrm{O}_{2}$ al 100\% a más de 2 atmósferas a nivel del mar para aumentar la presión parcial de oxígeno en el plasma, lo que permite aumen- 
tar la actividad de fibroblastos, producción de colágeno y angiogénesis. de manera concomitante aumenta la capacidad bactericida e inhibe la formación de toxinas. Se recomienda de 2.0 a 2.4 atmósferas durante 90 a 120 minutos una vez o dos veces al día. Su uso principal es en heridas crónicas o de difícil manejo ${ }^{28}$.

Recomendación: eficacia baja, nivel de evidencia II y III
8. Radioterapia: la adición de radiación a la extirpación quirúrgica disminuye las tasas de recurrencia de $1 \%$ a $35 \%$. La radiación daña los fibroblastos proliferantes directamente induciendo su apoptosis. Suele iniciarse 1 a 3 días después de la extirpación de la cicatriz, con una dosis total de 10 a 15 Gy, fraccionado en 5 a 6 sesiones. Contraindicado en niños y en áreas como mama y tiroides ${ }^{29,30}$.

Recomendación: eficacia alta, nivel de evidencia III.

\section{BIBLIOGRAFÍA}

1. Landis SJ. Chronic wound infection and antimicrobial use. Adv Skin Wound Care 2008; 21: 531-40.

2. Bowler PG, Duerden BI, Armstrong DG. Wound microbiology and associated approaches to wound management. Clin Microbiol Rev 2001: 14: 244-69

3. Sørensen LT. Wound healing and infection in surgery: the pathophysiological impact of smoking, smoking cessation, and nicotine replacement therapy: a systematic review. Ann Surg. 2012 Jun;255(6):1069-79

4. Yates C, May K, Hale T, Allard B, Rowlings N, Freeman A, et al. Wound chronicity, inpatient care, and chronic kidney disease predispose to MRSA infection in diabetic foot ulcers. Diabetes Care 2009; 32: 1907-9.

5. Russell $L$. The importance of patients' nutritional status in wound healing. Br J Nurs. 2001 Mar;10(6 Supp():S42, S44-9.

6. Dryden SV, Shoemaker WG, Kim JH. Wound management and nutrition for optimal wound healing. Atlas Oral Maxillofac Surg Clin North Am. 2013 Mar;21(1):37-47

7. Wicke C, Halliday B, Allen D, Roche NS, Scheuenstuhl H, Spencer MM et al. Effects of steroids and retinoids on wound healing. Arch Surg. 2000 Nov; 135(11):1265-70.

8. Gieringer M, Gosepath J, Naim R. Radiotherapy and wound healing: principles, management and prospects (review). Oncol Rep. 2011 Aug;26(2):299-307.

9. Tredget $E E$, Nedelec $B$, Scott PG, et al. Hypertrophic scars, keloids, and contractures. The cellular and molecular basis for therapy. Surg Clin North Am 1997;77(3):701-30.

10. Robles DT, Moore E, Draznin M, et al. Keloids: pathophysiology and management. Dermatol Online J 2007;13(3):9.

11. Draaijers LJ, Tempelman FR, Botman YA, Tuinebreijer WE, Middelkoop E, Kreis RW, et al. The patient and observer scar assessment scale: a reliable and feasible tool for scar evaluation. Plast Reconstr Surg. 2004 Jun;113(7):1960-5; discussion 1966-7.

12. Sullivan T, Smith J, Kermode J, et al. Rating the burn scar. J Burn Care Rehabil 1990; 11:256.

13. Bock O, SchmidOtt G, Malewski P, Mrowietz U. Quality of life of patients with keloid and hypertrophic scarring. Arch Dermatol Res 2006; 297:433.

14. Sidle DM, Kim H. Keloids: prevention and management. Facial Plast Surg Clin North Am. 2011 Aug;19(3):505-15,

15. Svensjö T, Pomahac $B$, Yao $F$, et al. Accelerated healing of full thickness skin wounds in a wet environment. Plast Reconstr Surg 2000; 106:602.

16. Muzaffar SJ, Pollock JC, Southern SJ. Vitamin E--an aid to wound healing? J Plast Reconstr Aesthet Surg. $2011 \mathrm{Jul}$;64(7):969; author reply $969-70$
17. Sclafani AP, Gordon L, Chadha M, Romo T 3rd. Prevention of earlobe keloid recurrence with postoperative corticosteroid injections versus radiation therapy: a randomized, prospective study and review of the literature. Dermatol Surg 1996; 22:569

18. Kiil J. Keloids treated with topical injections of triamcinolone acetonide (kenalog). Immediate and long term results. Scand J Plast Reconstr Surg 1977; 11:169.

19. Muneuchi G, Suzuki S, Onodera M, et al. Long term outcome of intralesional injection of triamcinolone acetonide for the treatment of keloid scars in Asian patients. Scand J Plast Reconstr Surg Hand Surg 2006; 40:111.

20. Wilson AM. Eradication of keloids: Surgical excision followed by a single injection of intralesional 5 fluorouracil and botulinum toxin. Can J Plast Surg 2013; 21:87.

21. Ledon JA, Savas J, Franca K, et al. Intralesional treatment for keloids and hypertrophic scars: a review. Dermatol Surg 2013; 39:1745

22. Payapvipapong K, Niumpradit N, Piriyanand C, Buranaphalin S, Nakakes $A$. The treatment of keloids and hypertrophic scars with intralesional bleomycin in skin of color. J Cosmet Dermatol. 2015 Mar;14(1):83-90.

23. Anzarut A, Olson J, Singh $P$, et al. The effectiveness of pressure garment therapy for the prevention of abnormal scarring after burn injury: a metaanalysis. J Plast Reconstr Aesthet Surg 2009; 62:77

24. Bran GM, Brom J, Hörmann K, Stuck BA. Auricular keloids: combined therapy with a new pressure device. Arch Facial Plast Surg 2012; 14:20.

25. Koike S, Akaishi S, Nagashima Y, et al. Nd:YAG Laser Treatment for Keloids and Hypertrophic Scars: An Analysis of 102 Cases. Plast Reconstr Surg Glob Open 2014; 2:e272

26. Jin R, Huang X, LiH, et al. Laser therapy for prevention and treatment of pathologic excessive scars. Plast Reconstr Surg 2013; 132:1747.

27. Marquardt Y, Amann PM, Heise R, Czaja K, Steiner T, Merk HF Characterization of a novel standardized human three-dimensional skin wound healing model using non-sequential fractional ultrapulsed CO2 laser treatments. Lasers Surg Med. 2015 Mar;47(3):257-65.

28. Mills BJ. Wound healing: the evidence for hyperbaric oxygen therapy. Br J Nurs. 2012 Nov 8-21;21(20):28, 30,32, 34.

29. Emad M, Omidvari S, Dastgheib L, et al. Surgical excision and immediate postoperative radiotherapy versus cryotherapy and intralesional steroids in the management of keloids: a prospective clinical trial. Med Princ Pract 2010; 19:402.

30. Kim J, Lee SH. Therapeutic results and safety of postoperative radiotherapy for keloid after repeated Cesarean section in immediate postpartum period. Radiat Oncol J 2012; 30:49. 